\title{
Intracranial Inflammatory Pseudotumor: A Case Report
}

\author{
Manizhe Ataee Kachuei ${ }^{1,{ }^{*}}$ \\ ${ }^{1}$ Department of Radiology, Iran University of Medical Sciences, Tehran, Iran \\ "Corresponding author: Department of Radiology, Iran University of Medical Sciences, Tehran, Iran. Tel: +98-9132267198, Email: manizhe.ataei64@gmail.com
}

Received 2019 September 14; Revised 2020 September 21; Accepted 2021 March 15.

\begin{abstract}
\section{Introduction}

Inflammatory pseudotumor is a rare lesion that can affect any organ; however, it is more common in the lungs and orbits (1-3). Primary inflammatory pseudotumor of the central nervous system (CNS) is extremely rare. These lesions may be intra-axial, extra-axial, or a mix of them. Only few cases of intracranial inflammatory pseudotumor have been reported $(4,5)$. We report a case of intracranial inflammatory pseudotumor involving both brain parenchyma and dura.
\end{abstract}

Primary inflammatory pseudotumor of the central nervous system is extremely rare. These lesions may be intra-axial, extra-axial, or a mix of them. We report a case of intracranial inflammatory pseudotumor involving both brain parenchyma and dura.

Keywords: Intracranial, Magnetic Resonance Imaging, Inflammatory Pseudotumor

\section{Case Presentation}

The patient was a 50-year-old woman who presented with headache, blurred vision, and seizure over several months. Biochemical, hematological, and serological tests were unremarkable.

Magnetic resonance imaging (MRI) demonstrated a suprasellar, lobulated mass with an extension and adhesion to the base of the inferior portion of the frontal lobe and optic chiasm, encasing anterior cerebral arteries (ACA) bilaterally. The lesion appeared isointense on T1-weighted images and hyperintense on T2-weighted images with fairly central hypointense foci. Post-contrast images showed a strong contrast enhancement and dural tail mimicking meningioma or metastasis.

The patient underwent surgery, but the lesion could not be resected completely because of extreme adhesion to hypothalamus and optic chiasm, and so only partial resection was done.

Microscopic section of pathological examination revealed brain tissue, which was infiltrated by a moderate number of inflammatory cells such as lymphocytes and plasma cells around blood vessels. The small vessels showed irregular proliferation. The brain tissue was intact.

On immunohistochemical staining, the inflammatory cells were positive for CD38 and CD68, and only few cells were positive for IgG4. The perivascular lymphoplasmacytic infiltration was compatible with the inflammatory pseudotumor.

The patient's symptoms improved after partial resection. Informed consent was obtained from the patient for surgery and publication.

\section{Discussion}

Inflammatory pseudotumor is a rare lesion characterized by collagen fibers and non-neoplastic proliferation of inflammatory cells, especially plasma cells. These tumors are more common in the lungs and orbits, but other organs such as CNS and the gastrointestinal tract can also be involved but with less frequency $(4,6)$.

Intracranial occurrence of inflammatory pseudotumor is extremely rare (6-10). A review of 57 cases of intracranial inflammatory pseudotumor shows that most of these lesions are dural based (60\%) and pure intra-axial lesions are less common (12\%) (11).

In our case, the lesion was located as a mass-forming lesion attached to the inferior basal portion of frontal lobes and the suprasellar region with dural attachment and adhesion to optic chiasm, encasing ACAs bilaterally.

Inflammatory pseudotumor imaging findings are fairly similar regardless of which organ is involved. These lesions appear isointense to hypointense on T1-weighted images and fairly hypointense in T2-weighted images on MRI. Relative enhancement of tumors is noted on both MRI and computed tomography (CT) images $(1,2,12)$. 
In our case, the lesion was isointense on T1-weighted images and hyperintense on T2-weighted images, containing central hypointense foci and demonstrating strong enhancement after administration of contrast injection (Figures 1-3).

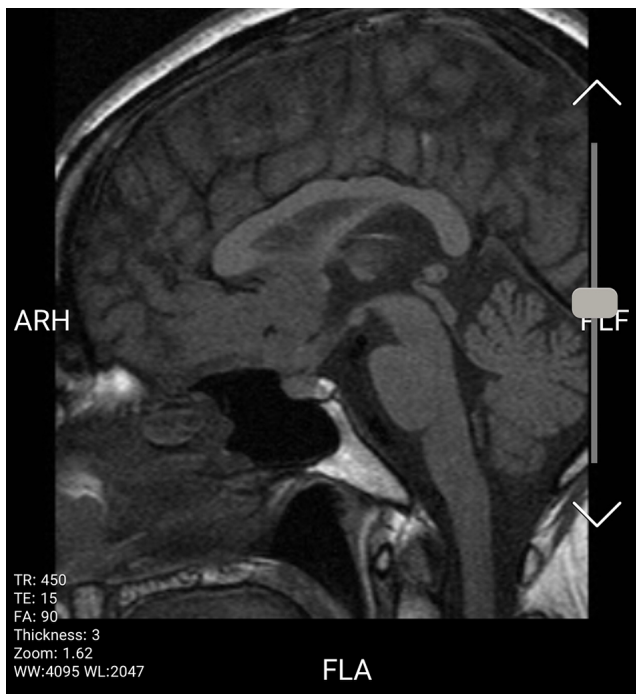

Figure 1. Sagital T1 image shows a lobulated isointense suprasellar mass with adhesion to base of inferior portion of frontal lobe and optic chiasm.

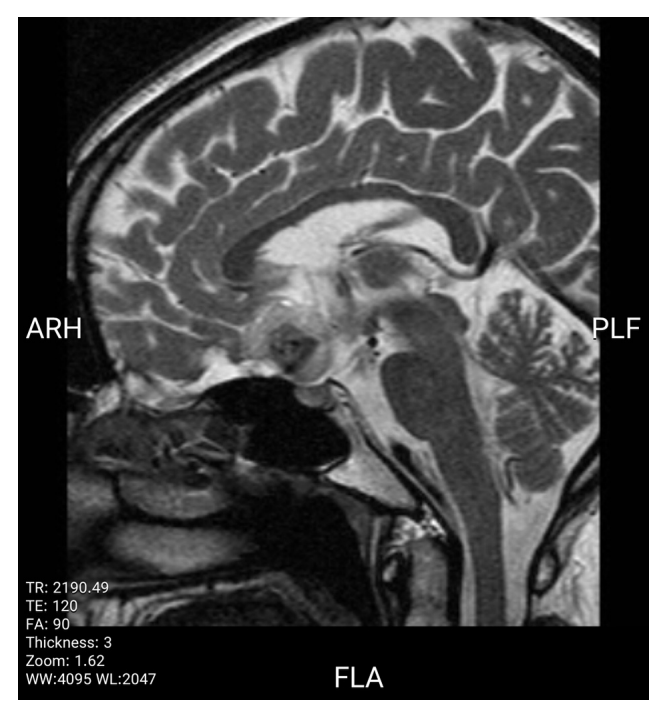

Figure 2. Sagital T2 image shows hyperintense suprasellar mass with fairly central hypointense foci.

Diagnosis was made by biopsy. Pathological examination revealed perivascular lymphoplasmacytic infiltration compatible with the inflammatory pseudotumor.

In conclusion, intracranial inflammatory pseudotu-

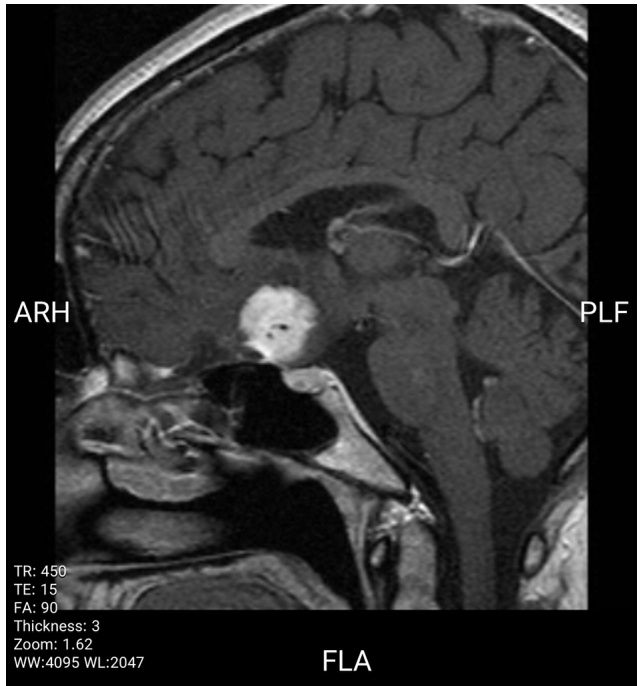

Figure 3. Sagital T1 post contrast shows strong enhancement of tumor and dura tale.

mors are rare and their imaging findings can be fairly similar to malignant tumors. Low-signal intensity on $\mathrm{T} 2$ images may be one of the most important characteristics of these tumors, enabling us to distinguish them from malignant tumors in appropriate clinical settings.

\section{Footnotes}

Authors' Contributions: AM, study concept and design, analysis and interpretation of data, drafting of the manuscript, critical revision of the manuscript for important intellectual content.

Conflict of Interests: The authors have no affiliation with any organization with a direct or indirect financial interest in the subject matter discussed in the manuscript.

Financial Disclosure: None declared.

Funding/Support: No funding or support was received for this study.

Informed Consent: Written informed consent was obtained.

\section{References}

1. Park $\mathrm{SB}$, Lee JH, Weon YC. Imaging findings of head and neck inflammatory pseudotumor. AJR Am J Roentgenol. 2009;193(4):1180-6. doi 10.2214/AJR.09.2398. [PubMed: 19770345].

2. Narla LD, Newman B, Spottswood SS, Narla S, Kolli R. Inflammatory pseudotumor. Radiographics. 2003;23(3):719-29. doi 10.1148/rg.233025073. [PubMed: 12740472].

3. Segawa Y, Yasumatsu R, Shiratsuchi H, Tamae A, Noda T, Yamamoto $\mathrm{H}$, et al. Inflammatory pseudotumor in head and neck. Auris Nasus Larynx. 2014;41(3):321-4. doi: 10.1016/j.anl.2013.11.002. [PubMed: 24359703]. 
4. Ishihara M, Izumoto S, Iwatsuki K, Yoshimine T. Immunohistochemical study of multiple inflammatory pseudotumors with both brain and spinal cord involvement-Case report. Neurol Med Chir (Tokyo). 2010;50(3):246-50. doi: 10.2176/nmc.50.246. [PubMed: 20339279].

5. Kilinc M, Erturk IO, Uysal H, Birler K, Evrenkaya T, Akkalyoncu BB. Multiple plasma cell granuloma of the central nervous system: A unique case with brain and spinal cord involvement. Case report and review of literature. Spinal Cord. 2002;40(4):203-6. doi: 10.1038/sj.sc.3101271. [PubMed: 11965561].

6. Kamisawa T, Okamoto A. IgG4-related sclerosing disease. World J Gastroenterol. 2008;14(25):3948-55. doi: 10.3748/wjg.14.3948. [PubMed 18609677]. [PubMed Central: PMC2725332].

7. Kasliwal MK, Suri A, Gupta DK, Suri V, Rishi A, Sharma BS. Sphenoid wing inflammatory pseudotumor mimicking a clinoida meningioma: Case report and review of the literature. Surg Neurol. 2008;70(5):509-13. doi: 10.1016/j.surneu.2007.07.075. [PubMed 18207558].
8. Lin YJ, Yang TM, Lin JW, Song MZ, Lee TC. Posterior fossa intracranial inflammatory pseudotumor: A case report and literature review. Surg Neurol. 2009;72(6):712-6. discussion 716. doi: 10.1016/j.surneu.2009.01.029. [PubMed: 19608242].

9. Pimentel J, Costa A, Tavora L. Inflammatory pseudotumor of the choroid plexus. Case report. J Neurosurg. 1993;79(6):939-42. doi: 10.3171/jns.1993.79.6.0939. [PubMed: 8246063].

10. Sitton JE, Harkin JC, Gerber MA. Intracranial inflammatory pseudotumor. Clin Neuropathol.1992;11(1):36-40. [PubMed: 1547580].

11. Hausler M, Schaade L, Ramaekers VT, Doenges M, Heimann G, Sellhaus B. Inflammatory pseudotumors of the central nervous system: Report of 3 cases and a literature review. Hum Pathol. 2003;34(3):253-62. doi: 10.1053/hupa.2003.35. [PubMed:12673560].

12. Nakayama K, Inoue Y, Aiba T, Kono K, Wakasa K, Yamada R. Unusual CT and MR findings of inflammatory pseudotumor in the parapharyngeal space: Case report. AJNR Am J Neuroradiol. 2001;22(7):1394-7. [PubMed: 11498435]. 